\title{
ASPECTOS COMPORTAMENTAIS DAS SÍNDROMES DE DOR CRÔNICA
}

\author{
JOSÉ TEOTONIO DE OLIVEIRA*
}

\begin{abstract}
RESUMO - O conhecimento dos mecanismos biológicos da dor não é suficiente para a compreensão das síndromes de dor crônica frequentemente encontradas pelos neurologistas, como lombalgias, cervicobraquialgias e mialgias, nas quais nenhuma anormalidade significativa é encontrada. Aspectos psicológicos, como distúrbios do humor e ansiedade, e aspectos sociais, como ganhos secundários do tipo aposentadorias e indenizações, podem ter papel relevante na iniciação e perpetuação dos sintomas. Fatores psicológicos e sociais produzem um comportamento de doença anormal, caracterizado basicamente por uma desproporção entre sinais objetivos escassos, queixas exacerbadas e alegação de incapacidade. A identificação de um comportamento de doença anormal, através da observação e avaliação dos comportamentos dos pacientes, é um método válido e útil para fins diagnósticos e quanto a natureza das queixas. O médico deve agir de modo a não reforçar as convicções organicistas dos pacientes, orientando-se quanto à propedêutica e tratamento pelos sinais objetivos e não pelo vigor das queixas e alegação de incapacidade. A sociedade deve também abster-se de adotar políticas que estimulam comportamentos de doente anormais.
\end{abstract}

PALAVRAS-CHAVE: dor crônica, doença, comportamento anormal.

\section{Behavioral aspects of chronic pain syndromes}

ABSTRACT - The knowledge of biological pain mechanisms are not sufficient for the understanding of patients with chronic pain syndromes such as low back, cervicobrachial and muscle pain. Psychological and psychosocial aspects play important roles in the setting and perpetuation of symptoms. Mood and anxiety disorders, secondary gains such as early retirement and financial compensations, must all be acknowledged by the physician as possible contributors to the symptoms. Abnormal illness behavior may better characterize patients with chronic pain syndromes. Behavior observation, which is akin to medical practice, is therefore a powerfull tool in the diagnosis and management of these syndromes. Physicians ought be very careful in not reinforcing the patients already strong organic convictions regarding their symptoms, avoiding making decisions based on patients complaints and alleged disabilities, and assigning poorly defined and disputable diagnosis labels. Society needs also to refrain from policies that encourage abnormall illness behaviors.

KEY WORDS: chronic pain, abnormal illness behavior.

O diagnóstico e o manejo de pacientes com sintomas sem patologia evidente é difícil e a maioria dos médicos não recebe educação e treinamento nesta tarefa ${ }^{1}$, sendo o médico treinado para procurar sempre uma patologia ou disfunção fisiológica responsável pelos sintomas. Por outro lado, explicações não-orgânicas não são aceitas por grande parte dos pacientes ${ }^{2-6}$. Estes fatores levam a uma demanda de rótulos, tanto por médicos quanto por pacientes, de diagnósticos que sugiram bases orgânicas dos sintomas. Diagnósticos surgem, de modo geral associados a algum descobrimento científico mais recente, e desaparecem quando as evidências científicas contrárias tornam-se avassaladoras ${ }^{2,4}$.

*Departamento de Psiquiatria e Neurologia da Faculdade de Medicina da Universidade Federal de Minas Gerais (UFMG), Serviço de Neurologia e Neurocirurgia do Hospital das Clínicas da UFMG. Aceite: 6janeiro-2000.

Dr. José Teotonio de Oliveira - Av. Pasteur, 89/1107 - 30150-290 Belo Horizonte MG - Brasil. 
As síndromes de dor crônica (SDC) sem patologias, com as quais o neurologista está em contato frequente, incluem as cefaléias, lombalgias, cervicobraquialgias (incluída aqui a LER, lesão por esforço repetitivo) e as mialgias. Em muitas destas síndromes os agentes nocivos não são demonstráveis e, mesmo assim, as pessoas acometidas alegam grau elevado de sofrimento e incapacidade, ficando o médico perplexo pela desproporção entre queixas e sinais objetivos. $\mathrm{O}$ aumento da prevalência destas queixas, principalmente relacionadas ao trabalho, em uma época de melhoria acentuada das condições de trabalho e do aumento da longevidade, é também intrigante ${ }^{7}$. Apesar da maioria dos pacientes se verem como medicamente doentes e serem grandes utilizadores de serviços $\operatorname{médicos}^{8}$, eles mantêm uma evolução clínica notavelmente estável através dos anos ${ }^{9}$.

Outro motivo de perplexidade e frustração do médico em relação aos pacientes com SDC da época atual é a resistência destes às explicações tranquilizadoras e aos tratamentos paliativos. Estas resistências são provavelmente devidas ao declínio da autoridade e prestígio do médico, à interferência sensacionalista e emocional da mídia e aos interesses de indivíduos e organizações de várias naturezas na manutenção das síndromes ${ }^{10,11}$.

O conhecimento dos mecanismos biológicos da dor não é suficiente para a compreensão dos pacientes com dores crônicas, sendo necessário a avaliação dos aspectos psicológicos e sociais envolvidos, que é o objetivo desta revisão.

\section{TAXONOMIA}

As SDC não têm marcadores biológicos, sendo os diagnósticos baseados em queixas. Sinais clínicos atribuídos a algumas delas, como na fibromialgia e síndrome do desfiladeiro torácico, são geralmente de natureza duvidosa ou então de especificidade baixa ${ }^{12-15}$. A superposição entre as diversas síndromes é comum. Em 70,9\% dos casos diagnosticados como LER pode ser feito o diagnóstico de fibromialgia ${ }^{16}$. Setenta por cento dos pacientes de fibromialgia e $30 \%$ dos pacientes de sensibilidade química múltipla preencheram também os critérios diagnósticos para síndrome de fadiga crônica ${ }^{17}$. A tentativa de compartimentalizar os sintomas em síndromes diferentes torna-se um exercício de "riscar linhas na água" ${ }^{18}$. Os diferentes rótulos diagnósticos aplicados refletem mais a área de interesse do examinador do que algum critério científico.

As SDC podem ser classificadas de acordo com a região acometida: cervicobraquialgia, lombalgia, fibromialgia, cefaléia, etc. Nesta classificação não há compromisso com etiologia ou patologia.

Algumas classificações envolvem etiologias e mecanismos patogênicos, mesmo que estes sejam controversos: lesão por esforços repetitivos, lesão por traumas repetitivos, síndrome de uso excessivo, fibromialgia, distrofia simpática reflexa. A sintomatologia dolorosa pode ser atribuída a alguma patologia localizada, como em síndrome do desfiladeiro torácico, síndrome do pronador redondo, tenossinovite, etc.

Outras classificações são baseadas em aspectos psicológicos. Em algumas, predominam as descrições fenomenológicas, enquanto em outras são realçados aspectos sociológicos.

O Diagnostic and Statistical Manual IV (DSM-IV) ${ }^{19}$ classifica as síndromes dolorosas crônicas entre os transtornos somatoformes, transtornos factícios e a simulação. A dor também pode ser manifestação de doença psiquiátrica, como depressão, ansiedade e psicose.

Nos transtornos somatoformes a dor pode fazer parte do transtorno de somatização, quando é acompanhada de sintomas gastrointestinais, sintoma sexual e sintomas pseudoneurológicos. Quando a dor é o único sintoma, é classificada como transtorno doloroso. Os transtornos somatoformes são considerados de natureza inconsciente e involuntários, ao contrário dos transtornos factícios e simulação, que são conscientes e voluntários. Somatização é diagnosticada quando a pessoa apresenta 
padrão de queixas somáticas múltiplas, recorrentes e importantes clinicamente, a ponto de haver necessidade de um tratamento. Deve haver história de dor relacionada a, pelo menos, quatro locais. Deve haver também história de, pelo menos, dois sintomas gastrointestinais que não dor ${ }^{19}$.

Dor é a mais comum das formas de apresentação de conversão ${ }^{20}$. Reação de conversão (também conhecida como histeria) é caracterizada pela presença de sintomas ou deficits afetando a função motora ou sensitiva voluntária, que simulam uma condição neurológica ou outra condição médica geral $^{19}$. Os sintomas conversivos são chamados de pseudoneurológicos por estarem relacionados ao funcionamento motor ou sensitivo voluntário. Os sintomas motores e sensitivos conversivos são diagnosticados pela sua variabilidade e distribuição que não se enquadram em padrões anatômicos e fisiológicos conhecidos. Estes sintomas ocorreriam porque a pessoa deseja obter algum ganho, financeiro ou interpessoal. Os sintomas da reação de conversão são precipitados por conflitos ou outros fatores tensionais ${ }^{20}$.

O diagnóstico de reação de conversão não é feito pela simples exclusão de uma doença orgânica ou por características de personalidade. Pode ser feito clinicamente, como em várias outras doenças médicas, diagnosticadas exclusivamente em bases clínicas, sem exames comprobatórios. $\mathrm{Na}$ simulação os sintomas e exame são iguais aos que ocorrem na conversão. Se houver acentuada discrepância entre o sofrimento ou deficiência alegados pela pessoa e os achados objetivos e falta de cooperação durante a avaliação diagnóstica, deve-se suspeitar de simulação ${ }^{19}$. O diagnóstico de simulação fundamenta-se na identificação de um ganho externo ou secundário (em relação a um ganho interno, como no transtorno factício), como a principal motivação para o comportamento ${ }^{21}$. Na simulação, se os fatos situacionais são conhecidos, o observador pode discernir a motivação do comportamento. As motivações comuns são escape de uma situação nociva (p.ex., prisão), monetárias (p.ex., indenizações), ou a obtenção de narcóticos (na forma de analgésicos ou sedativos).

O transtorno factício difere de conversão e simulação porque os sintomas e os sinais são produzidos conscientemente mas a motivação é inconsciente. O objetivo, na maioria das vezes, é obter assistência e atenção médica através de hospitalização. O ganho visado no transtorno factício é puramente psicológico ${ }^{21}$.

A dificuldade dos diagnósticos descritos acima é determinar se os sintomas são produzidos consciente ou inconscientemente. Naturalmente, a confissão de fabricação de sintomas ou a observação de funcionamento normal em situações desconhecidas pela pessoa, são provas indiscutíveis de que os sintomas são voluntários, mas esta situação é rara. Um comportamento pode ser considerado consciente quando o indivíduo escolhe os momentos e locais para agir de modo a parecer doente, e toma decisões de encobrimento, o que seria demonstração de controle dos sintomas ${ }^{22}$. O paciente que sonega ou acrescenta dados para obter licença médica é claramente um simulador. A decisão de se obter dados, como um exame alterado que poderá significar a concessão de um benefício, é indicação de que o paciente está agindo conscientemente. Somatizadores e simuladores abastecemse de sintomas comuns da vida diária como fadiga, dor de cabeça, dor nas costas, dolorimentos musculares, tonteiras, os quais, para a maioria das pessoas, não são causas suficientes para levá-las a procurar assistência médica ${ }^{10}$. Dor músculo-esquelética, associada a fadiga, é comum em pessoas normais, independentemente do tipo de atividade ${ }^{23,24}$. Em um estudo epidemiológico, $36,7 \%$ das pessoas relataram terem sofrido dor articular, $31,5 \%$ dor lombar e $24,3 \%$ dor na perna ${ }^{25}$. Outra fonte de sintomas são as doenças da moda, como brucelose crônica, alergia a alimentos, infecção pelo vírus Epstein-Barr, hipoglicemia e, no momento atual, lesão por esforços repetitivos e fibromialgia, entre inúmeras outras ${ }^{2-4}$.

\section{VISÃO SOCIOLÓGICA}

O comportamento é a manifestação dos elementos psicológicos e sociológicos e tem a vantagem de poder ser facilmente observado, tornando-o muito útil na prática médica. A prática proporciona 
aos médicos oportunidade de observar os comportamentos das pessoas em situações de doenças e avaliar o grau de adequação de dado comportamento, não devendo o médico abdicar deste conhecimento. A observação de um comportamento, e emissão de juízo de valor a respeito dele, é um método válido e, aliás, necessário e amplamente utilizado para a formulação de diagnósticos e condutas.

As sociedades reservam aos indivíduos doentes um papel de doente, em que a pessoa tem direitos e deveres. Os direitos são a liberação da responsabilidade pela situação de incapacidade em que se encontra e a liberação das obrigações com a sociedade, a qual aceita arcar com os ônus. Por outro lado, a pessoa fica obrigada a reconhecer que o papel de doente é indesejável, procurar ajuda médica e a abandonar o papel de doente logo que a condição causadora desapareça ${ }^{26-29}$. As pessoas que assumem o papel de doente apresentam o comportamento de doença.

Comportamento de doença são as várias percepções, pensamentos, sentimentos e atos que influenciam o significado pessoal e social dos sintomas, incapacidade e suas conseqüências ${ }^{26,30}$. Quando o comportamento de doença é desproporcional ao nível de doença presente é considerado anormal $^{27,28}$, podendo ocorrer maximização ou minimização da doença em relação aos dados objetivos.

Os comportamentos desenvolvidos pelos doentes podem se estóicos e controlados, histriônicos e dramáticos e a comunicação pode ser verbal ou tomar a forma de disfunção física. Em relação à afetividade, a pessoa pode estar deprimida, ansiosa ou indiferente. Nas relações com outros ela pode ser hostil, desconfiada, temerosa, implorante ou excessivamente cooperativa e agradável ${ }^{27}$.

No intuito de convencer a sociedade e garantir o papel de doente, o indivíduo amplia os sintomas e reforça as crenças, utilizando informações adquiridas no seu ambiente próximo (casa, local de trabalho) e, cada vez mais, através da imprensa e de grupos de interesses ${ }^{10}$. Desta maneira, constrói-se um conjunto de sintomas e atitudes que possam garantir legitimamente o papel de doente com seus benefícios inerentes. O comportamento de doença anormal pode ser também induzido ou reforçado por médicos, por atribuição de sintomas a patologias vagas, investigações e tratamentos excessivos ${ }^{1,31}$.

O comportamento de doença anormal é desenvolvido como estratégia de comunicação ou meio de adaptação $0^{32-34}$ e para obter ganhos financeiros ${ }^{33,35}$.

Segundo Pilowsky ${ }^{27}$, comportamento de doença anormal é o termo que melhor descreve os pacientes com dor crônica sem patologia, sendo melhor do que os termos transtorno somatoforme, histeria ou reação de conversão, porque chama a atenção para as dimensões comportamentais, psicológicas e sociológicas. Pilowsky e Spence ${ }^{32}$ desenvolveram um questionário de comportamento de doença nos quais são avaliados 7 fatores: hipocondria, convicção de doença, focalização somática ou psicológica, distúrbio afetivo, inibição afetiva, negação e irritabilidade. Este questionário foi aplicado em pacientes com dor crônica intratável, cuja queixa era desproporcional à patologia, e a pacientes também com sintomas dolorosos proeminentes freqüentando clínicas de reumatologia, radioterapia, pulmonar e fisioterapia. Os resultados revelaram que convicção de doença e ausência de resposta a informações tranquilizadoras do médico caracterizam os pacientes com dor crônica desproporcional à patologia $\mathrm{a}^{28,32,36}$.

Embora a faixa de comportamento de doença normal talvez não seja possível de ser determinada exatamente, a experiência do médico, baseada em dados pessoais e históricos do doente, permite avaliar razoavelmente quando o comportamento está dentro do esperado.

O conceito de comportamento de doença anormal evita a necessidade de se determinar se os sintomas são produzidos consciente ou inconscientemente.

\section{COMO DETECTAR A ETIOLOGIA NÃO-ORGÂNICA DE SINTOMAS}

O diagnóstico de não-organicidade de um sintoma deve ser feito cautelosamente, não deve ser baseado somente na ausência de sinais orgânicos, pois várias doenças somáticas não têm sinais 
orgânicos. Para se fazer o diagnóstico do que Meador ${ }^{37}$ chamou de não-doença, exige-se profundo conhecimento da doença. Não-doença seria a doença que a pessoa acha que tem.

Quando queixas verbais não são corroboradas pelos fatos clínicos, é obrigação dos médicos obter informações a respeito da pessoa do paciente. Tais informações freqüentemente não são fornecidas voluntariamente. Nos contextos de doença ocupacional e em situações que não resultam de acidente ou lesão claramente definidas ou documentadas, além das situações judiciais, deve-se obter informações de fontes colaterai ${ }^{38,39}$. Em situações legais, entre as quais as situações ocupacionais, os reclamantes podem exagerar ou mesmo falsificar incapacidades, de livre arbítrio ou instruídos por terceiros. O contato intenso com advogados, médicos e outros reclamantes, proporciona treinamento excelente a respeito das alegadas lesões e incapacidade ${ }^{38}$. Em outras palavras, as pessoas estão muito mais motivadas para mentir do que para cooperar com a recuperação, já que esta milita contra os seus interesses. Enganar os médicos não é tarefa difícil porque eles são educados para acreditar na sinceridade das pessoas ${ }^{38,39}$.

Entre os sinais que sugerem uma natureza não-orgânica de sintomas estão "la belle indifference", alterações sensitivas não-anatômicas, alterações sensitivas que variam em exames repetidos, alterações de sinais motores e sensitivos pela sugestão, hemianestesia com transição exatamente na linha mediana, perda de sensibilidade vibratória unilateralmente quando os 2 lados da testa ou do esterno são testados; fraqueza em padrão de cessão súbita ${ }^{19,40}$, variabilidade de posturas e fraquezas com distração $0^{41}$, reações exageradas (choro, gritos, sustos, tensionamento muscular, sudorese, síncope ${ }^{28,41,42}$, uso exagerado de aparelhos ortopédicos ${ }^{42}$, descrições detalhadas e repetitivas da situação causadora da lesão alegada ${ }^{42}$, queixas de negligência e descaso do sistema médico e previdenciário, discrepância entre queixas, incapacidade e sinais objetivos, falta de cooperação ${ }^{42}$. Os pacientes com síndromes de dor crônica têm convicção elevada de terem um problema orgânico e são também caracteristicamente resistentes às explicações tranqüilizadoras dadas pelos médicos ${ }^{28,32,36}$.

Nenhum dos sinais e comportamentos descritos é absoluto e específico, podendo ocorrer em doenças orgânicas ${ }^{40,43} \mathrm{e}$, alguns, são pouco sensíveis, tornando ainda mais árdua a tarefa de diferenciar doenças orgânicas de distúrbios não-orgânicos e de simulação, especialmente em situações de confronto.

\section{CONCLUSÕES}

A avaliação de pessoas com queixas de dor crônica sem sinais objetivos continua uma tarefa difícil, apesar dos avanços dos conhecimentos sobre os mecanismos nociceptivos. Na avaliação destes pacientes o médico deve estar atento para os aspectos psicológicos e psicossociais associados à percepção de dor, bem como a todas as questões não-médicas, como interesses financeiros dos reclamantes e seus patrocinadores. A avaliação de dores crônicas não pode ficar restrita aos aspectos puramente biológicos. O comportamento do doente deve ser cuidadosamente observado e avaliado e as discrepâncias devidamente registradas.

O médico não pode deixar que a sua conduta relativa a exames e tratamento seja dirigida pelo grau ou veemência das queixas, devendo guiar-se, primordialmente, por sinais objetivos.

Havendo dificuldades em se definir exatamente os processos envolvidos, é mais sensato usar denominações sintomáticas, sem compromisso com etiologias patogêneses.

Ao reconhecer o papel do comportamento nas SDC, fica claro que a abordagem destes pacientes só será eficaz se promover uma mudança nos fatores que levam ao comportamento indesejado. Esta mudança dependerá da ação do médico na sua relação com os pacientes individualmente bem como de ações da sociedade, como a retirada de estímulos para o prolongamento ou adoção do papel de doente. A dor crônica atualmente, principalmente quando associada a alto grau de incapacidade, não é mais exclusivamente um problema médico. 


\section{REFERÊNCIAS}

1. Loeser JD, Sullivan M. Doctors, diagnosis, and disability: a disastrous diversion. Clin Orthop 1997; 336:61-66.

2. Shorter E. From paralysis to fatigue: a history of psychosomatic illness in the modern era. New York: Free Press, 1992.

3. Shorter E. The borderland between neurology and history:conversion reactions. Neurol Clin 1995;13:229-239.

4. Shorter E. Somatization and chronic pain in historic perspective. Clin Orth 1997;336:52-60.

5. McWhinney IR, Epstein RM, Freeman. Rethinking somatization. Ann Intern Med 1997;126:747-750.

6. Wessely S. Chronic fatigue syndrome: a 20th century illness? Scand J Work Environ Health 1997;23(Suppl 3):17-34

7. Allan DB, Waddell G. An historic perspective on low back pain and disability. Acta Orthop Scand 1989;60(Suppl 234):1-23.

8. Katon W, von Korff M, Lin E, et al. Distressed high utilizers of medical care. DSM-III-R diagnoses and treatment needs. Gen Hosp Psychiatry 1990;12:355-362.

9. Smith GR, Monson RA, Ray DC. Patients with multiple unexplained symptoms: their characteristics, functional health and health care utilization. Arch Intern Med 1986;146:69-72.

10. Barsky AJ, Borus JF. Somatization and medicalization in the era of managed care. JAMA 1995; 274:1931-1934.

11. Barsky AJ, Borus JF. Functional somatic syndromes. Ann Intern Med 1999; 130: 910-921.

12. Bohr TW. Fibromyalgia syndrome and myofascial pain syndrome: do they exist? Neurol Clin 1995;13:365-384.

13. Bohr T. Problems with myofascial syndrome and fibromyalgia syndrome. Neurology 1996;46:593-597.

14. Cherrington M, Cherrington C. Thoracic outlet syndrome: reimbursement patterns and patient profiles. Neurology 1992;42:943-945.

15. Wilbourne AJ. Thoracic outlet syndrome is overdiagnosed. Muscle Nerve 1999;22:130-136.

16. Helfenstein M, Feldman D. Prevalência da síndrome de fibromialgia em pacientes diagnosticados como portadores de lesão por esforços repetitivos (LER). Rev Bras Reumatol 1998;38:71-77.

17. Buchwald D, Garrit D. Comparison of patients with chronic fatigue syndrome, fibromyalgia, and multiple chemical sensitivities. Arch Intern Med 1994;154:2049-2053.

18. Shorter E. On the nature and origin of psychosomatic symptoms. Scand J Work Environ Health 1997;23(Suppl 3):105-112.

19. Diagnostic and statistical manual of mental disorders. 4Ed. Washington DC: American Psychiatric Association, 1994.

20. Weintraub MI. Chronic pain in litigation: real or imagined? $47^{\text {th }}$ Annual Meeting, American Academy of Neurology, San Francisco, 1995:440-23 - 440-28.

21. Eisendrath SJ. Psychiatric aspects of chronic pain. Neurology 1995; 45(Suppl 9):26-34.

22. Teasell RW, Shapiro AP. Strategic-behavioral intervention in the treatment of chronic nonorganic motor disorders. Am J Physical Med Rehabil 1994;73:44-50.

23. Hadler NM. Cumulative trauma disorders. J Occup Med 1990;32:38-41.

24. Cunningham LS, Kelsey JL. Epidemiology of musculoskeletal impairments and associated disability. Am J Public Health 1984;74:574-579.

25. Kroenke K, Price RX. Symptoms in the community: prevalence, classification, and psychiatric commorbity. Arch Intern Med 1993;153:2474-2480.

26. Mechanic D. The concept of illness behavior. J Chron Dis 1962;15:189-195.

27. Pilowsky I. Abnormal illness behavior. Br J Med Psychol 1969;42:347-351.

28. Waddell G, Pilowsky I, Bond MR. Clinical assesment and interpretation of abnormal illness behavior in low back pain. Pain 1989;39:41-53.

29. Fordyce WE. On the nature of illness and disability. Clin Orthop 1997;336:47-51.

30. Mechanic D. Illness behavior, social adaptation, and the management of illness. J Nervous Mental Dis 1977;165:79-87.

31. Koyanou K, Pither CE, Wessely S. Iatrogenic factors in chronic pain. Psychosomatic Med 1997;59:597-604.

32. Pilowsky I, Spence ND. Patterns of illness behavior in patients with intractable pain. J Psychosomatic Res 1975;19:279-287.

33. Ford CV. Illness as a lifestyle: the role of somatization in medical practice. Spine 1992;17:S338-S343.

34. Ford CV. Somatization and fashionable diagnosis: illness as a way of life. Scand J Work Environ Health 1997; 23(Suppl 3):7-16.

35. Ford CV. Dimensions of somatization and hypochondriasis. Neurol Clin 1995;13:241-253.

36. Pilowsky I, Spence ND. Pain and illness behaviour: a comparative study. J Psychosomatic Res 1976;20:131-134

37. Meador CK. The art of nondisease. New Engl J Med 1955;272:92-95.

38. Faust D. The detection of deception. Neurol Clin 1995;13:255-265.

39. Hall HV, Pritchard DA. Detecting malingering and deception. Delray Beach, Florida: St. Lucie Press, 1996.

40. Gould R, Miller BL, Goldberg MA, Benson DF. The validity of hysterical signs and symptoms. J Nerv Ment Dis 1986;174:593-597.

41. Waddell G, Finlayson D, Main CJ. Symptoms and signs; physical disease or illness behavior? Br Med J 1984;289:739-741.

42. Wilbourne AJ. The electrodiagnostic examination with hysteria-conversion reaction and malingering. Neurol Clin 1995;13:385-404.

43. Cunnien AJ. Psychiatric and medical symptoms associated with deception. In Rogers R (eds). Clinical assesment of malingering and deception. London: Guilford Press, 1997:23-46. 